\title{
La précision de la méthode thermodynamique à la lumière de deux années d'application industrielle
}

\section{The accuracy of the thermodynamic method as seen after two years of industrial application}

\author{
PAR G. WILTM \\ INGÉNEUR A I,E.D.F. \\ DIVISION TECHNIQUE GÉNÉRALE
}

\begin{abstract}
Rappel des principes de la méthode thermométrique de mesure des rendements des turbines hydrauliques : description de l'appareillage. - Etude analytique de sept causes d'erreurs. - Enumération des précautions à apporter dans les techniques opératoires pour sauvegarder la précision des mesures. - Comparaison des sources d'errears et de lear palliatifs dans le cas des mesures thermométriques et des mesures aux moulinets; examen de la valeur des essais en valeur absolue et comparativement avec les méthodes classique; exposé de divers cas singuliers.
\end{abstract}

Recapitulation of the principles of the thermometrical method of measuring the efficiency of hydraulic turbines: description of equipment. Analytic study of the seven causes of error. Precautions to be taken when applying the method so as to mainiain accuracy of measurements. Comparison of sontces of error and the remedies for them in thermometrical measurements; examination of the absolute value of tests and their value when compared with classic methods; an account of various singular cases.
Le principe de la méthode thermométrique de mesure des rendements des turbines hydrauliques, imaginé par M. Porrson en 1914, est bien connu; on sait qu'il était basé sur l'équivalence des pertes de la turbine et de l'échauffement de l'eau turbinée.

Plusieurs modalités d'application devaient par la suite être imaginées par M. Porrson et M. Barbillon; un certain nombre d'expérimentateurs habiles, au premier rang desquels il faut citer - outre évidemment M. Porrson lui-même - M. Fontaine et M. Volle, mesurant au moyen d'un thermomètre à mercure au $1 / 100^{\circ}$ de degré la température de l'eau du canal de fuite et celle d'une eau prélevée dans la conduite forcée et détendue sans travail jusqu'à la pression atmo- sphérique, obtenaient des résultats encourageants. Nous ne reviendrons pas sur ces essais qui ont fait l'objet de plusieurs publications; cependant, la comparaison des rendements ainsi obtenus et de ceux obtenus par les méthodes classiques mettait l'accent sur la nécessité d'une mise au point de la technique aussi bien que de la théorie si l'on voulait espérer obtenir une précision seulement comparable à celle des moulinets.

En 1952, le Service de la Production Hydraulique d'Electricité de France, directement intéressé à la question, chargeait sa «Division Technique Générale 》 d'étudier le problème.

Les résultats de cette étude, guidée par M. Ferry et à laquelle M. Campmas prit une part 
importante, firent en 1954 l'objet dans la Houille Blanche (1) d'un article où étaient exposées une étude théorique et la description d'un dispositif expérimental, notablement différent de celui appliqué jusqu'alors et justifié par des considérations aussi bien d'utilisation industrielle que de précision. Des comparaisons entre des résultats « thermométriques » et ceux obtenus par les méthodes "classiques » terminaient l'exposé.

Mon propos aujourd'hui n'est pas de revenir sur cette publication, mais de rapporter le fruit de deux années d'application au stade industriel, en insistant un peu sur les causes d'erreurs et les moyens pratiques que l'expérience nous a appris à mettre en œuvre pour les détecter et les pallier.

\section{Principe}

Je rappellerai cependant très brièvement les deux aspects théoriques et pratiques du problème.

Le passage d'une certaine masse d'eau d'un état à un autre, définis chacun par une pression, une vitesse, une température, constitue une transformation obéissant obligatoirement à la loi sur la conservation de l'énergie. Une masse unité d'eau subit au cours d'une telle transformation une variation: d'une part de son énergie hydraulique, d'autre part de son énergie interne définie à partir de sa température et de son volume. Nous appelons variation d'énergie totale la somme des variations de l'énergie hydraulique et interne et le principe de la conservation de l'énergie indique que cette variation est égale au travail fourni sur l'arbre. Ceci peut s'exprimer par les formules de base ci-après (fig. 1)

Formules de base

Energie hydraulique.....g H $=g\left(\frac{\gamma 2}{2 g}+\frac{\mathrm{P}}{\bar{\omega}}+z\right)$

Energie interne........

Energie totale.........

Puissance développée sur $\mathrm{IV}^{r}$

l'arbre par unité de débit $Q$

Loi de conservation de $\Delta(u+g \mathrm{H})=\frac{\mathrm{W}}{\mathrm{Q}}$
l'énergie $\ldots \ldots \ldots \ldots \ldots \ldots$

Expression du rendement. $n=\frac{\mathrm{W}}{g \mathrm{Q} \mathrm{H}_{n}}$

$$
=\frac{\Delta(u+g \mathbf{H})}{g \mathbf{H}_{n}}
$$

Fig. 1.

(1) - Juillet-Octobre 1954. qui donnent également l'expression du rendement de l'opération.

Le bilan de l'opération s'exprime en fonction des éléments suivants :

$1^{\circ}$ Ceux relatifs aux énergies mécanique et hydraulique, c'est-à-dire :

- Ia variation de pression, de cote, de vitesse, de poids spécifique, éventuellement la fourniture de travail;

$2^{\circ}$ Ceux relatifs à l'énergie interne $\ll \boldsymbol{u} »$, qui sont :

-. La transformation d'une partie de l'énergie mécanique ou hydraulique en chaleur;

- Le travail des forces extérieures par suite de la variation de volume spécifique (dans une turbine à vapeur, c'est là un terme prépondérant; dans une turbine hydraulique, c'est, du fait de la faible compressibilité de l'eau, un terme de second ordre) ;

- Une variation de température liée à la détente adiabatique de l'eau (cet élément thermodynamique, très important pour les gaz, est pour l'eau, en l'occurrence, loin d'être négligeable);

-- Eventuellement les échanges de chaleur avec les milieux extérieurs.

Le calcul de $\Delta u$ à partir des relations de la thermodynamique conduit à l'introduction d'un terme correctif dont l'expression complète figure sur l'article précité de la Houille Blanche; il faut retenir que le refroidissement adiabaticue y représente la part de beaucoup la plus importante. Pour les pressions usuelles, et avec les modalités que nous avons adoptées, l'incidence du terme correctif sur le rendement est nulle ou négligeable aux environs de 3 à 4 , el atteint $4 \%$ pour des températures de l'eau de $15^{\circ}$.

Ia figure 2 donne le schéma expérimental sous sa forme la plus générale, suffisamment explicite par Jui-même, el l'expression du rendement, également sous sa forme la plus générale.

On voit sur cette formule figurer, outre le terme correctif thermodynamique $\alpha_{1}$, un terme $\beta_{\text {c }}$ qui intervient dans l'expression de la hauteur de chute. Ce terme n'est pas propre au caractère thermodynamique de la méthode; il est exclusivement mécanique ef ne prend en compte que les variations de volume spécifique de l'eau. Sa valeur est faible, elle croît avec la hauteur de chute pour atteindre $0,5 \%$ dans le cas de très hautes chutes.

On voit que plusieurs modalités expérimentales correspondant à des simplifications sont possibles; on peut tout d'abord faire $H_{1}=0$, c'esta-dire faire porter la mesure sur l'eau détendue 


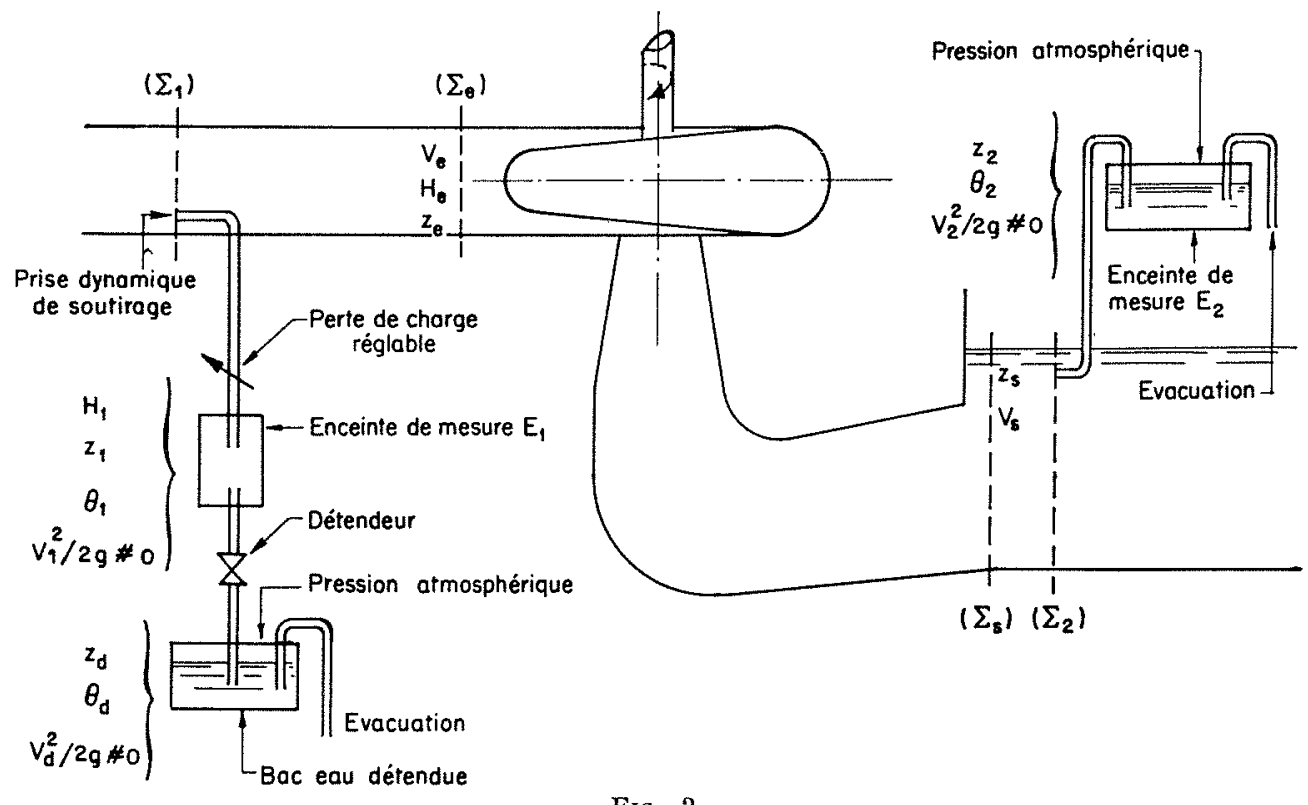

FIg. 2.

Méthode thermodynamique. - Schéma de principe.

$$
\eta=\frac{\mathrm{H}_{u}}{\mathrm{H}_{n}}=\frac{\mathrm{H}_{1}\left(1-\alpha_{1}\right)+a\left(\theta_{1}-\theta_{2}\right)+z_{1}-z_{2}}{\mathbf{H}_{e}\left(1-\beta_{e}\right)+\left(\mathrm{V}_{e}^{2}-\mathrm{V}_{s}^{2}\right) / 2 g+z_{e}-z_{s}}
$$

à la pression atmosphérique : c'est ce que faisait M. PoIrson qui mesurait $\theta_{1}$ et $\theta_{2}$. Ses mesures n'étaient pas faussées par la méconnaissance du terme $\alpha_{1}$ dont le rôle n'a été pressenti que beaucoup plus récemment.

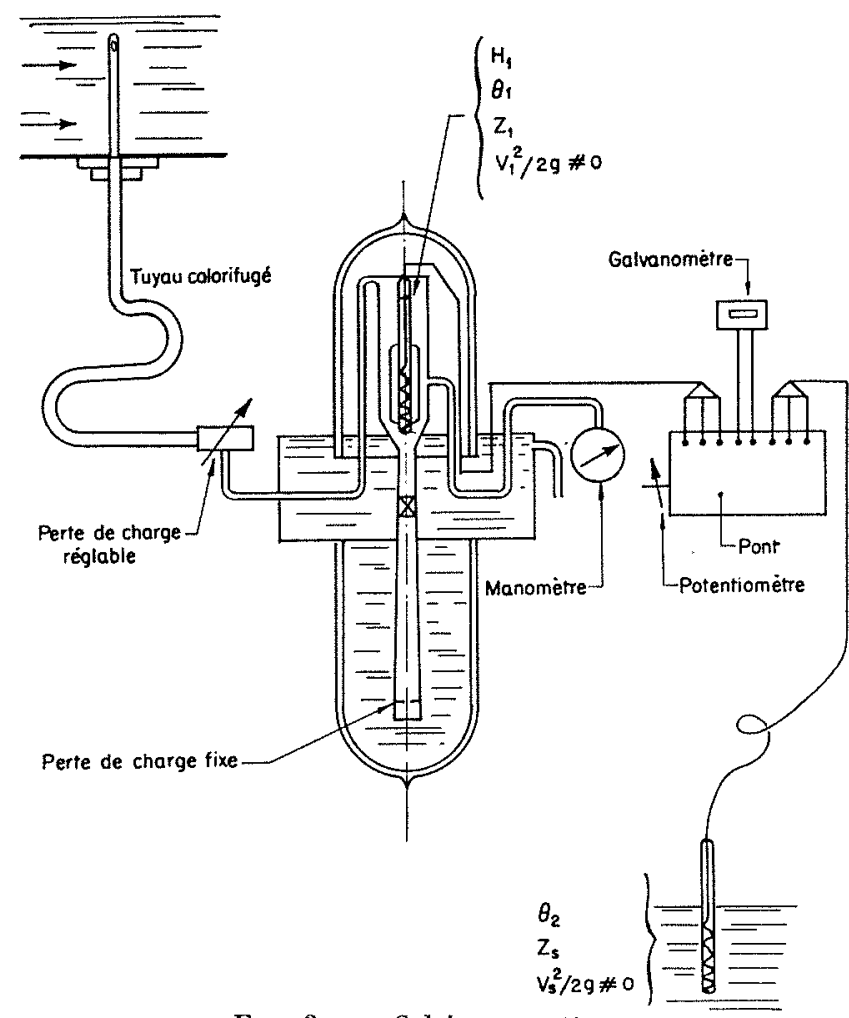

FIG. 3. - Schéma pratique.
La mesure de la température avec la précision exigée restant une opération très délicate (l'équivalence de $10^{-3^{\circ}} \mathrm{C}$ exprimée en hauteur de chute est en effet de $42,7 \mathrm{~cm}$ ), nous avons recours à un autre procédé consistant à annuler $\theta_{1}-\theta_{2}$; nous faisons subir à l'eau prélevée dans la conduite une transformation dont les thermodynamiciens diraient qu'elle est à enthalpie constante; par le jeu de la détente variable, on transforme une partie de l'énergie mécanique en chaleur. Cette détente partielle est dosée de façon à obtenir l'égalité de la température $\theta_{1}$ avec celle du canal de fuite : la mesure des températures est remplacée par une vérification de leur égalité, ce qui est incomparablement plus facile.

De plus, l'enceinte où se fait la mesure de $\theta_{1}$ et $\mathrm{H}_{1}$ est dimensionnée de telle sorte que $\mathrm{V}_{1}$ soit négligeable (la figure 3 donne le schéma de principe de l'appareillage).

\section{Erreurs}

Sous cette forme, la méthode thermométrique - ou plutôt thermodynamique - tout en conservant la grande simplicité d'emploi qui constitue un de ses meilleurs atouts, s'affirme comme remarquablement précise quoique à vrai dire les sources d'erreurs soient nombreuses; celles-ci sont dues à des phénomènes parasites qui peuvent venir se superposer aux phénomènes de base énumérés tout à l'heure et auraient pu faire 
craindre à un moment donné que la méthode ne reste une vue de l'esprit.

Nous allons reprendre l'analyse de ces causes d'erreur's à la lumière de l'expérience acquise depuis la rédaction de l'article de la Houille Blanche. Disons tout de suite que nos recherches ont montré que non seulement leur incidence resle faible, mais qu'il est possible soit de les éviter, soit d'introduire des corrections mesurées ou calculées. Par ailleurs, aucune objection autre que celles se rattachant aux causes d'imprécision que nous allons examiner ne nous a été faite sur le principe ou la mise en cuvre du procédé.

On pourrait les classer en cinq catégories :

- une certaine incertitude sur les valeurs numériques exactes de $\alpha_{1}$;

- Ies dissolutions de gaz et les changements d'étal;

- les échanges thermiques avec l'extérieur;

- - les irrégularités de répartition de la température ou de l'énergie dans les sections de mesure;

- les variations de température de l'eau dans le temps.

\section{Calculs DES $\alpha_{1}$}

$\alpha_{1}$ est calculé à partir des constantes physiques de l'eau, en particulier les coefficients de compressibilité et de dilatation. Or, la précision avec laquelle ces coefficients sont connus est peut-être insuffisante, du fait en particulier que ce sont leurs dérivées qui interviennent. Cependant, l'incidence de $\alpha_{1}$ sur le rendement dépasse rarement $3 \%$. On est fondé à penser que les valeurs numériques de $\alpha_{1}$ sont exactes à $10 \%$ près, d'où une erreur systématique possible de $0,3 \%$ sur le rendement. Il y aurait évidemment intérêt à resserrer cette marge. L'expérimentation qui permettrait ce résultat est prévue, mais reste à mettre en cuvre.

\section{Dissolution}

Nous ne parlerons que pour mémoire des dissolutions ou désorptions de gaz; la correction est approximativement de $0,15.10^{-30} \mathrm{C}$, soit $6,5 \mathrm{~cm}$ par cm" d'air à pression atmosphéricque par litre d'eau. Ce chiffre varie d'ailleurs peu avec la nature du gaz. Les dégazages que nous avons eu l'occasion d'observer n'étaient jamais assez importants pour justifier une correction.

\section{Cavitation}

La cavitation à la sortie d'une Francis constitue un cas de changement d'état. Nous avons eu l'accasion de citer (1) un exemple de prélè-

(1) - La Houille Blanche, op, cit. vement aval sous la roue, dans la zone de cavitation, entrainant un rendement notoirement excessif. L'explication du phénomène était cue la formation de vapeur refroidissail l'eau; du fait de la différence de masse spécifique des deux fluides: eau et vapeur, le soutirage dynamique n'en conservait pas les proportions, l'échantillonnage était incorrect. Le rendement de cette mème turbine, mesuré dans des conditions habituelles, c'est-à-dire la section de mesure étant en aval dans le canal de fuite, a donné des résultats normaux.

Nous n'avons pas de nouvel exemple à citer montrant l'influence de la cavitation; mais il est rigoureux de dire que si l'eau ne présente pas d'échanges d'énergie avec l'extérieur pendant son trajet dans l'aspirateur, son niveau d'énergie totale ne varie plus dès la sortie de la roue; la refermeture des bulles de cavitation fait disparaître la cause de l'erreur.

\section{ECHANGES DE CHALEUR}

Dans le domaine des échanges de chaleur avec l'extérieur, il faut distinguer ceux qui intéressent l'appareillage et ceux relatifs à la machine. Les premiers, malgré toutes les précautions prises, ne sont pas toujours négligeables. On définit facilement le terme correctif en mesurant un même rendement avec deux débits de soutirage franchement différents, par exemple 0,2 et 0,4 $1 / \mathrm{s}$. Pour notre appareillage standard, et dans des conditions normales de mesure, l'ordre de grandeur de l'erreur est $2.10^{-3^{\circ}} \mathrm{C}$ pour un débit soutiré de $0,4 \mathrm{l} / \mathrm{s}$; pour une chute d'une centaine de mètres, Ia correction atteint dans ces conditions - $0,8 \%$; elle peut devenir négligeable pour des chutes supérieures. Cependant, certains montages nécessités par des conditions locales peuvent entraîner des corrections sensiblement plus importantes (1).

Remarquons qu'il n'y a pas lieu d'attacher une importance excessive à ces échanges qui peuvent être tolérés, à condition de les mesurer, ce qui, on l'a vu, est facile.

Le problème se pose différemment pour les échanges thermiques entre l'eau de la turbine et l'extérieur' il n'existe pas de moyen commode

(1) Il faut observer à ce sujet que les apports de calories ne sont certainement pas exclusivement lies aux echanges entre l'appareillage et l'air ambiant; nous avons été amenés à penser que des apports non négligeables pouvaient avoir leur origine dans les échauffements nés tres localement des pertes de charge de l'ecoulement principal au contact du dispositif de soutirage (on sait en effet que les pertes de charge dans le circuit de soutirage lui-même ne peuvent etre mises en cause). Enfin, dans certains cas, l'écoulement de l'eau dans la conduite forcée peut mettre en vibration la sonde de soutirage, et il semble qu'une partie de l'énergie mécanique ainsi mise en jeu soit entraînéc sous forme de chaleur par l'eau soutiréc. 
de les mesurer; mais les calculs auxquels on peut se livrer en utilisant les coefficients classiques de transmission de chaleur les montrent négligeables. Nous nous croyons, dans le cas général, fondés à admettre l'absence d'erreur sensible de ce fait, et ce d'autant plus que les faibles débits, plus favorables à une erreur sur la température, correspondent en général aux installations de haute chute, où l'incidence de cette erreur sur le rendement est plus faible, et inversement.

\section{AfrRation DES ROUES}

Dans un même ordre d'idées, l'aération des turbines peut être à l'origine d'apports de calories. Il est facile de calculer l'erreur commise, une fois calculé le débit d'air admis.

Deux sortes de phénomènes sont à considérer :

- Transfert de calories;

- Condensations ou vaporisations.

Ces deux effets se combinent, mais restent toujours faibles et tendent dans les cas habituels à se compenser. C'est ainsi que dans l'exemple suivant : eau à $10^{\circ}$, air à $15^{\circ}$, hygrométrie $50 \%$, l'erreur est nulle. Par contre, elle serait, avec les mêmes températures mais avec une hygrométrie de 100, et pour un débit d'air égal au débit d'eau (cas de certaines Pelton), de $+3,5 \cdot 10^{-3^{\circ}} \mathrm{C}$. De toute façon, il n'y a pas à proprement parler de problème tant que la proportion air/eau est inférieure à $1 / 10$, et une solution radicale consiste à couper l'aération, ce qui peut presque toujours se faire temporairement sans inconvénients.

Observons de plus que, pour un contrôle différentiel des rendements, l'incidence de termes correctifs éventuels est encore diminuée.

\section{RÉPARTITION DE L'ÉNERGIE}

Par contre, une cause sensible d'imprécision peut avoir son origine dans des irrégularités de répartition de l'énergie dans les sections de mesure. C'est ainsi que si la section aval est placée trop près de la turbine (moins de $5 \mathrm{~m}$ par exemple), la prospection dans cette section fait en règle générale apparaître, suivant le point considéré, des différences de rendement qui peuvent atteindre $1 \%$. Dans de tels cas, et s'il s'agit de mesures absolues, on peut se contenter, pour obtenir une valeur très approchée, d'une moyenne des rendements obtenus' en plusieurs points répartis dans la section. Ces défauts d'homogénéité sont liés au fonctionnement même de la turbine, ils se modifient légèrement avec la charge, mais on peut considérer que la réparti- tion se retrouve à charge égale identique d'un essai au suivant; au pire, on pourra choisir deux emplacements de mesures dans la section aval; on pourra vérifier que les hétérogénéités se conservent d'un essai à l'autre.

II est certain cependant que nous cherchons dans la mesure du possible à choisir la section aval à une distance de la turbine telle que l'homogénéité soit satisfaisante; des gradients sensibles n'ont jamais été observés à $15 \mathrm{~m}$ de la turbine. Dans certaines usines en projet, nous prévoyons un dispositif permanent de herses de soutirage effectuant automatiquement une moyenne approximative, et placé dans une section à distance optimum.

Voici, à titre d'exemple (fig. 4) la répartition des écarts de température traduits en écarts de rendement par rapport au rendement au centre

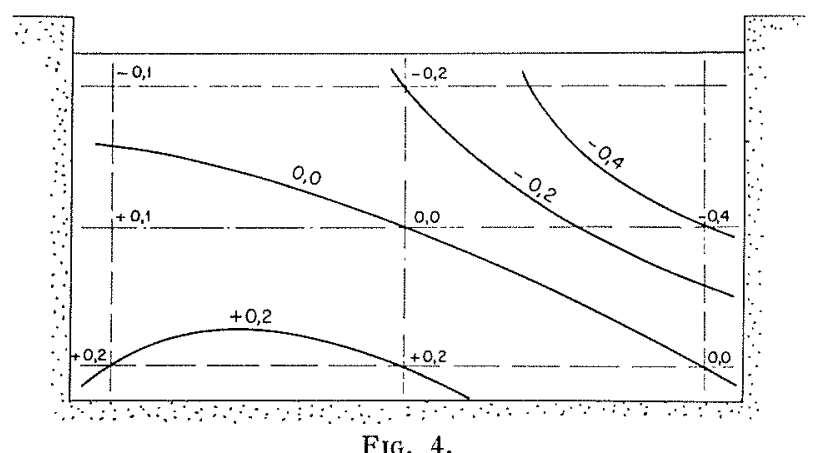

Fig. 4.

Hétérogénéité du rendement dans le canal de fuite. Pelton 2 jets; $\mathrm{H}_{n}=625 \mathrm{~m} ; \mathrm{P}=30.000 \mathrm{~kW}$

dans une section d'un canal de fuite placé à 2 ou $3 \mathrm{~m}$ en aval de la fosse d'une roue Pelton 2 jets, $35.000 \mathrm{~kW}, 650 \mathrm{~m}$. On observe une certaine dissymétrie et la présence systématique d'une eau plus chaude en surface, correspondant sans doute à l'entraînement des pertes par ventilation de la roue. Dans ce cas, nous avons effectué la moyenne, et on peut penser que la précision des mesures n'a pas été altérée de plus de quelques pour mille. De fait, les rendements ainsi relevés pour les roues identiques et pratiquement neuves de cette usine ne s'écartent pas les uns des autres de plus de deux pour mille.

En ce qui concerne les hétérogénéités dans la conduite, jusqu'à présent les essais de prospection suivant un diamètre n'ont rien montré de systématique, et les écarts constatés sont à la limite des grandeur's mesurables. Un soutirage sous une chute de $240 \mathrm{~m}$, fait à dessein dans un sillage important, a montré, par comparaison avec un soutirage dans le vif de l'écoulement, un écart maximum de 0,4 point, ce qui confirme l'hypothèse suivant laquelle l'énergie totale est pratiquement constante dans une section et, dans une large mesure, indépendante de la répartition de l'énergic hydraulique, sauf peut-être au 
voisinage immédiat de la paroi du fait du transfert de calories entre l'air ambiant et l'eau de la conduite. Mais cette énergie parasite représente localement une proportion trop faible de l'énergie globale pour qu'il soit, en règle générale, utile d'en tenir compte; il suffit de ne pas soutirer dans cette zone.

\section{INSTABILITÉ DE $\theta$ DANS ILE TEMPS}

Nous arrivons à la dernière cause d'erreurs, à vrai dire la plus gênante : l'instabilité des températures dans le temps. Une telle variation entraîne une erreur du fait que l'eau met un certain temps à passer de la section de mesure amont à la section aval; également, les jauges à résistance peuvent avoir des constantes de temps différentes. La correction est proportionnelle à la variation de température par unité de temps, et inversement proportionnelle au débit (approché) de la turbine. Elle peut se calculer, mais il est aussi simple d'obtenir le coefficient de proportionnalité en mesurant un rendement de la turbine sous une charge quelconque à un moment où la variation de température est nulle, puis à nouveau à un moment où cette variation a une certaine valeur.

Voici un graphique (fig. 5) récapitulant des essais faits il $y$ a plus d'un an dans le but de

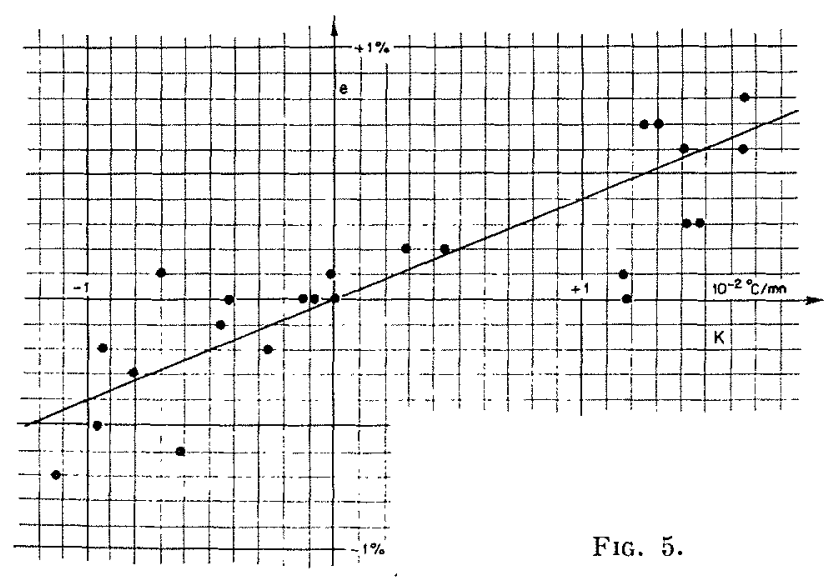

Corrélation entre l'erreur et la variation de température pour l'ouverture maximum.

comparer deux profils de roue, dans des condilions tout à fait défavorables, du fait en particulier des variations importantes de la tempéralure dans le temps, atteignant $10^{-2}{ }^{\circ} \mathrm{C} / \mathrm{min}$; du nuage de points, on peut tirer un coefficient de correction. Mais on constate en outre que la dispersion est beaucoup plus importante pour les variations rapides que pour les lentes. L'écart maximum, toute correction faite, atteint $\pm 0,4 \%$ dans le premier cas et $\pm 0,1$ dans le second. La plus grande partie de cette dispersion secondaire peut être attribuée au fait que le $d \theta / d t$ luimême est d'autant plus mal défini en valeur absolue qu'il est plus important.

La figure suivante (fig. 6) montre la courbe de rendement obtenue dans la même usine après correction des variations de température sur la base du graphique précédent. On constate que la

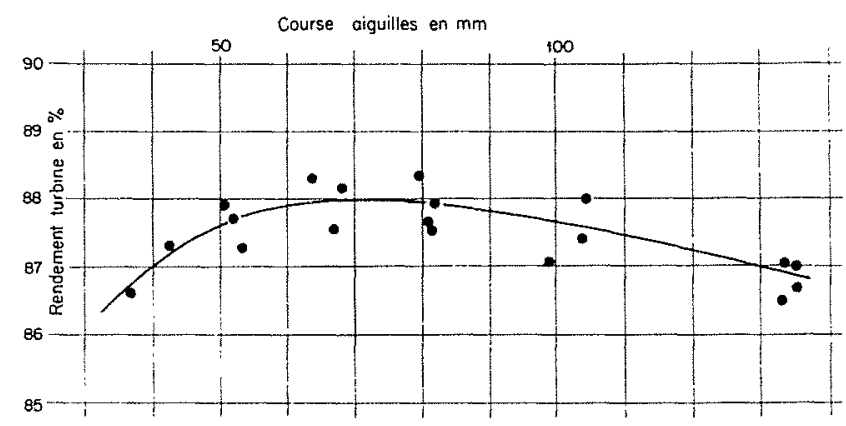

Fig. 6.

Turbine Pelton; $\mathrm{H}_{n}=360 \mathrm{~m}$.

dispersion des points individuels est au maximum de $\pm 0,5 \%$, soit une marge d'erreur accidentelle de $\pm 0,02$ à 0,3 point pour les charges comportant 3 ou 4 mesures.

Nous insistons bien sur le fait qu'il s'agit là d'un essai réalisé dans des conditions très défavorables : variations importantes de température allant jusqu'à $1 / 100$ de ${ }^{\circ} \mathrm{C}$ à la minute, alors que normalement nous ne tolérons que $5.10^{-3}$; appareillage et technique périmés. Enfin, alimentation des injecteurs par des conduites forcées séparées sur tout leur parcours, et réunies seulement à leur partie inférieure, de telle sorte que la bonne homogénéisation des températures $n$ 'avait pas le temps de se faire completement.

Voici donc passées en revue les causes d'erreurs qui peuvent altérer la précision de la méthode thermodynamique.

\section{Techniques opératoires}

On pourrait garder de cette énumération l'impression que ces mesures sont extrêmement délicates et que l'on est à la merci de la moindre inattention de l'opérateur. Ceci n'est pas tout à fait vrai, car cette énumération des causes d'erreurs est pratiquement limitative; la précision des mesures est effectivement sauvegardée par l'observation des cinq précautions općratoires suivantes :

- Vérification, par une prospection rapide, de l'homogénéité des températures dans le canal de fuite;

- Contrôle de l'évolution des températures de l'eau au cours des essais;

- Mesure des apports parasites de calories dans l'appareillage; 
- Vérification de la stabilité du pont de mesures thermique;

- Contrôle du dégazage éventuel.

Ces cinq contrôles élémentaires font systématiquement partie de la routine de toute opération de mesure de rendement. D'autres éléments de réussite résident en quelques détails pratiques qui, s'ils paraissent secondaires, n'en contribuent pas moins à augmenter dans une forte proportion la commodité et la précision de la méthode.

C'est d'abord la mise au point pour la mesure des pressions d'un dispositif dit « presse automatique », qui ajoute à la précision bien connue des manomètres à poids nort l'énorme avantage d'une lecture directe et instantanée, sans aucune manipulation (la précision d'un manomètre métallique du type Bourdon serait très insuffisante).

Ensuite (fig. 7), au lieu de chercher à réaliser l'égalité rigoureuse des températures et de lire la pression de détente partielle correspondante, ce qui constitue un travail pénible et presque

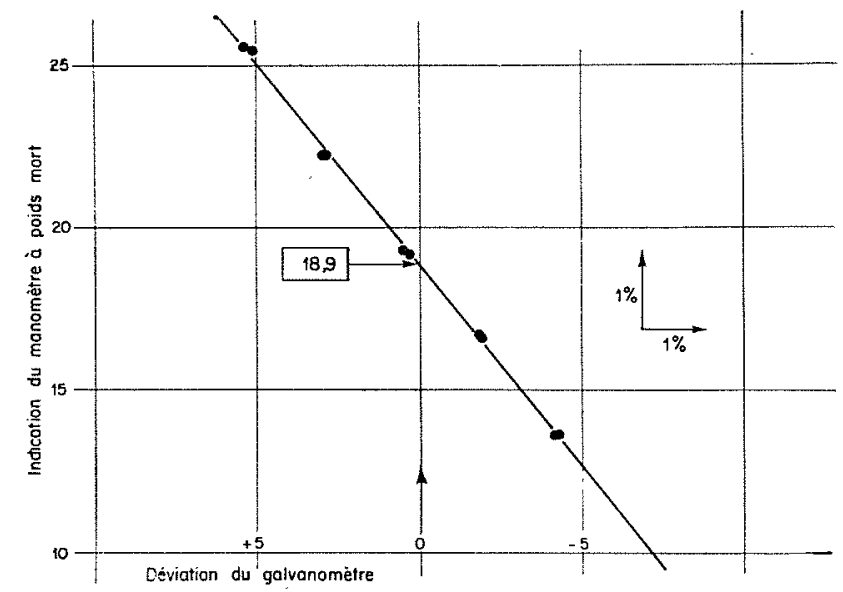

FIG. 7 .

Francis double horizontale : $\mathrm{H}_{n}=310 \mathrm{~m}$. Graphique des variations.

impossible si les pressions sont fluctuantes, nous établissons la correspondance entre les déviations du galvanomètre et les pressions partielles, celles-ci étant échelonnées de facon à encadrer la valeur relative à l'équilibre des températures - les lectures au galvanomètre sont faites au dixième de division, ce qui représente $16 \mathrm{~cm}$ d'eau, les lectures à la presse automatique à $10 \mathrm{~cm}$ d'eau près. La pression correspondant à l'équilibre des températures est interpolée sur la droite moyenne. Les points peuvent être portés au fur et à mesure des lectures, ce qui permet de se rendre compte rapidement de la dispersion, et d'augmenter le nombre de lectures le cas échéant. Celles-ci sont rapides, mais il est bon d'attendre la stabilisation pendant une minute après chaque changement de pression partielle.
Dans le cas particulier, l'opérateur réitérait ses lectures et a fait cinc relevés indépendants. On voit qu'il était inutile en l'occurence d'en faire plus; la dispersion des points de part et d'autre de la droite moyenne est de l'ordre de 1 pour mille. Le graphique permet d'obtenir une valeur de rendement : on lit en ordonnées sur l'échelle arbitraire des pressions, la pression de détente partielle correspondant au zéro du galvanomètre, c'est-à-dire à l'égalité des températures. Sur cet exemple, une unité en ordonnée représente $1,28 \mathrm{~m}$ d'eau (à noter que, dans l'exemple choisi, le groupement des points est bon, mais non exceptionnel).

\section{Comparaisons}

Une comparaison des sources d'erreurs et de leurs palliatifs dans les cas des mesures thermométriques et des mesures aux moulinets ne serait pas, croyons-nous, à l'avantage de ces derniers, tout au moins pour des chutes de plus de $200 \mathrm{~m}$. Les mesures aux moulinets sont en effet sujettes à des erreurs dont les origines ne sont pas toujours évidentes : tarage, freinage des hélices, influence du profil de la perche, mauvaise fixation ou vibrations de celles-ci, rotation de la veine d'eau, extrapolation des vitesses aux parois, incidence des mesures électriques, moins précises qu'on ne l'imagine bien souvent.

C'est pour ces motifs que les codes d'essais prévoient une tolérance sur la précision de $\pm 2 \%$. S'il est juste de reconnaître que, habituellement, tout laisse à penser que l'on reste dans ces limites, nous pourrions par contre citer des cas où cette marge a été notoirement dépassée.

Pour la méthode thermométrique, de nombreuses vérifications et réitérations nous ont montré que la marge d'imprécision qui est (dans des conditions satisfaisantes) de l'ordre de $1 \%$ aux environs de $100 \mathrm{~m}$, diminue rapidement pour des chutes plus élevées, jusqu'à atteindre 2 ou 3 dixièmes de point pour des chutes de l'ordre de $500 \mathrm{~m}$. Mieux : si les conditions sont défavorables, des tests permetlent d'en être averti et de fixer à priori la précision à atteindre, et, s'il le faut, de mieux choisir les conditions des essais : par exemple, sections de mesure, moment de la journée. Sil s'agit de contrôles périodiques, la qualité primordiale de la mesure est la fidélité; dans ce domaine, la supériorité de la méthode thermométrique ne peut que s'affirmer.

La question qui vient naturellement à l'esprit est celle de savoir ce que ralent ces essais en valeur absolue et en regard de ceux effectués par les méthodes classiques. Un certain nombre de comparaisons ont été faites, l'article précité de la Houille Blanche en donne quelques exemples; l'écart est, en règle très générale, inférieur à 
$1 \%$, ce qui est très satisfaisant pour les différentes catégories de méthodes.

Cependant, il est quelques cas où cet écart a atteint ou même dépassé $2 \%$, dans un sens ou dans l'autre; ce sont évidemment les plus intéressants à étudier et, effectivement, chaque fois que le cas s'est présenté, nous avons recherché l'origine du désaccord : en règle générale, nous n'avons pu mettre en évidence que des probabilités plus ou moins fortes en faveur de l'une des méthodes. Un rappel même bref de l'argumentation dans chaque cas particulier sortirait du cadre restreint de cet exposé.

Je relaterai cependant les divergences les plus importantes constatées : sur une turbine des Pyrénées ( $230 \mathrm{~m}, 7.500 \mathrm{~kW})$, deux essais aux moulinets, le deuxième constituant la réitération de l'autre, donment un rendement environ 3 points plus faible que deux essais thermodynamiques effectués à quelques mois d'écart. Le désaccord est ici absolument formel, et à priori, on ne saurait attribuer à une méthode plus de crédit qu'à l'autre. Cependant, la méthode thermodynamique a été exécutée dans de très bonnes conditions, les dispersions sont faibles. Côté moulinets, on observe une anomalie de répartition des vitesses; par ailleurs, les résultats moulinets feraient ressortir un défaut de similitude entre modèle et turbine assez surprenant, surtout en ce qui concerne la loi débit/ouverture - aux dires du constructeur qui a étudié la question de très près. Inversement, la similitude est beaucoup plus satisfaisante avec les essais thermodynamiques.

Le rendement d'une turbine du Massif Central $(125 \mathrm{~m}, 20.000 \mathrm{~kW})$ a été trouvé par la méthode thermodynamique de 5 points inférieur aux es sais classiques de réception. Mais ces derniers résultats étaient depuis l'origine considérés comme sujets à caution par le service qui avait exécuté les mesures lui-même, et les résultats thermodynamiques n'ont fait que confirmer un doute déjà ancien, sans toutefois qu'un indice quelconque ait jamais permis de retrouver l'origine de l'erreur.

Un cas assez récent concerne deux Pelton des Pyrénées $(410 \mathrm{~m}, 8.500 \mathrm{~kW}$ par roue). Le désaccord ici est relativement faible, mais systématique : les moulinets et un Rehbock se trouvent à plusieurs reprises d'accord pour attribuer aux roues un rendement d'environ $2 \%$ supérieur aux garanties du constructeur, alor's que le rendement thermodynamique « colle » à tròs peu de chose près avec la garantie.

Je ne m'arrêterai pas à l'argument suivant lequel il est plus probable que le rendement réel soit voisin des garanties et non $2 \%$ plus élevé. L'accord entre monlinets et Rehbock, assez troublant à première vue, peut être fortuit : on sait en effet que la formule de Rehbock a plutôt ten- dance à sous-estimer les débits, et par ailleurs, la vitesse de l'eau dans la section du canal de fuite où étaient placés les moulinets était un peu faible pour ceux-ci : $50 \mathrm{~cm} / \mathrm{s}$ environ à pleine charge; on constate effectivement que le désaccord, négligeable aux fortes charges, augmente très nettement quand la charge diminue. De son côté, la méthode thermodynamique a été appliquée ici dans de bonnes conditions, et absolument aucune difficulté particulière d'exécution ne pourrait expliquer une erreur de $2 \%$.

Rien n'exclut enfin que le désaccord ne porte sur la mesure des hauteurs de chute; sous ce rapport, la méthode thermodynamique n'est pas spécialement favorisée, quoique nous prenions des précautions supplémentaires pour que la précision avec laquelle est défini cet élément soit comparable à la précision de la mesure thermodynamique proprement dite (c'est ainsi que la chute nette est souvent mesurée simultanément par la pression totale et par la hauteur piézométrique majorée du $\mathrm{V}^{2} / 2 g$ ).

Je terminerai ce chapitre en citant un dernier cas de désaccord d'ailleurs bénin : 1,5\%, constaté à l'occasion de la mesure récente de rendement d'une pompe, quoique les valeurs des hauteurs de refoulement (environ $90 \mathrm{~m}$ ) aient été prises les mêmes pour toutes les méthodes (moulinets, diaphragme et thermodynamique); ici, la faible valeur de la hauteur de chute et l'emplacement de la section de mesure aval trop près de la sortie de la pompe, dans une zone où les énergies se trouvaient inégalement réparties, constituent évidemment des facteurs très défavorables à la précision des mesures thermodynamiques. Il est amusant toutefois de constater le fait suivant: sur une deuxième roue identique à la précédente, le constructeur avait particulièrement soigné la qualité de l'exécution et le poli des surfaces, escomptant ainsi une amélioration sensible du rendement. Or, les mesures faites cette fois exclusivement par les procédés classiques ont donné un rendement inférieur de 1 à 2 points au précédent, et ont done rejoint les valeurs thermodynamiques antérieures. Qu'en conclure? Que les améliorations apportées à la roue ont diminué son rendement ou, au contraire, que sur la premiere série d'essais, les résultats thermodynamiques étaient plus près de la vérité que les résultats des méthodes classiques? Il faudrait que nous refassions nos essais; le manque de temps, les difficultés d'accès et l'éloignement de la station, nous en ont jusqu'à présent empêchés.

J'aurais aimé m'étendre un peu longuement sur les applications de la méthode en cours d'exploitation, et en particulier sur le contrôle périodique de l'évolution des rendements dans le temps; un tel contrôle, outre des renseignements d'ordre général sur la résistance à l'usure des 
alliages employés, permet seul de déterminer sur des bases économiques objectives l'époque optimum du remplacement ou de la recharge

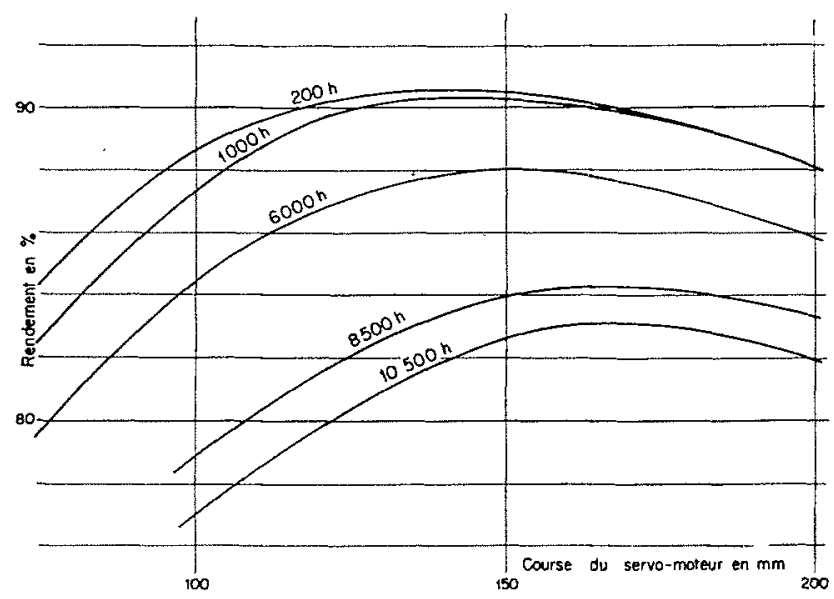

Fig. 8.

Rendement des turbines $27.300 \mathrm{~kW} ; \mathrm{H}_{n}=310 \mathrm{~m}$.

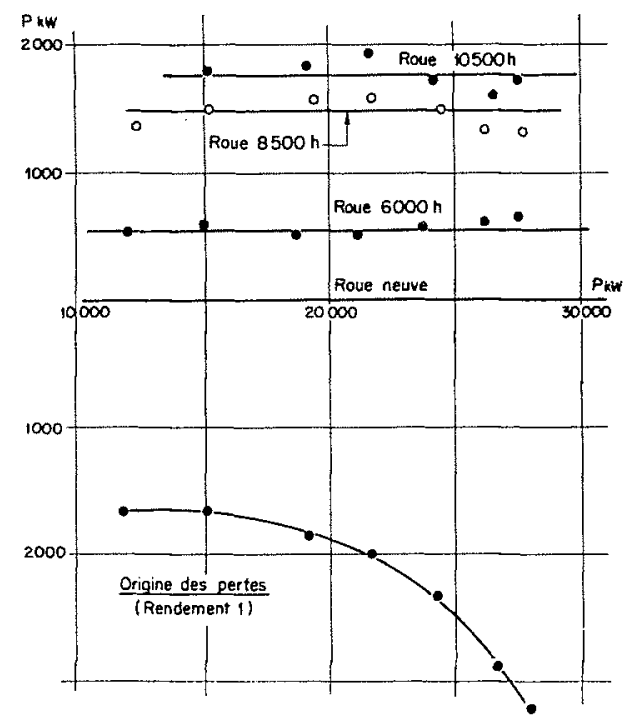

Fig. 9.

Pertes de puissance. Francis double, sans ceinture $\mathrm{H}_{n}=300 \mathrm{~m} ; \mathrm{P}=26.000 \mathrm{~kW}$

d'une roue ou d'un injecteur. L'exemple que j'ai retenu parmi beaucoup d'autres concerne une turbine Francis double sans ceinture, $27.000 \mathrm{~kW}$, $310 \mathrm{~m}$ de hauteur de chute. La figure 8 montre le rendement d'une turbine neuve et après environ $1.000,6.000,8.000$ et $10.000 \mathrm{~h}$ de fonctionnement. La figure suivante présente le même résultat, sous une forme un peu différente (fig. 9) faisant apparaître la perte de puissance entre l'état neuf et l'état usé. On voit que cette perte de puissance, pour une usure donnée, est une constante qui ne dépend pas de la charge de la machine. Unue intégration simple permet de chiffrer la perte d'énergie en fonction du temps. On trouve donc que l'usure a fait perdre pendant la première année de fonctionnement $1,6.10^{6} \mathrm{kWh}$, et $5 \mathrm{GWh}$ rien que pendant les six mois suivants. En mars prochain, celte roue aura deux ans et on peut escompter que la perte atteindra $13 \mathrm{GWh}$ (1). Un certain nombre d'enseignements peut être tiré de l'étude de celte évolution dans le temps, étude qui n'aurait sans doute pas été entreprise, ni surtout suivie de façon aussi serrée, s'il avait fallu recourir aux procédés classiques.

Le temps me manque pour parler également de l'application de la methode à la comparaison de tracés de roues, d'augets ou d'injecteurs, ou d'une façon générale de toute modification dans les formes ou les dispositions d'une turbine, domaine dans lequel aussi nous avons déjà rícolté de nombreux et utiles renseignements.

Il est certain que nous possédons là un moyen exceptionnel qui, s'ajoutant à d'autres procédés tels que la mesure des pertes aux labyrinthes et l'exploration des écoulements à la sortie des roues Francis dues à M. Fontaine, et depuis peu l'étude des phénomènes dynamiques au cours des charges critiques, nous donne les moyens de mieux analyser le travail réel de l'eau et, le cas échéant, de l'améliorer.

Nous signalerons pour terminer que les Etablissements Neyrpic ont entrepris la fabrication de notre matériel thermométrique sous une forme qui bénéficiera à la fois des plus récents perfectionnements et d'une présentation plus évoluée. Il sera possible ainsi de répondre à des demandes émanant en grande partie de l'étranger (Suisse et Italie) et de garder ainsi à cette technique son caractère bien francais.

\section{DISCUSSION}

(Président : M. Gariel)

M. le Président loue la probité scientifque de l'exposé de $M$. WILLM et considère qu'on a franchi anjourd'hui une grande étape dans l'application de la méthode thermométrique.

Sur la demande de M. Hupner, M. Willm précise les pourcentages des diverses corrections susceptibles d'intervenir normalement :

$$
\begin{aligned}
& \text { - appareillage : } \leqslant 1 \% ; \\
& \text { - répartition de l'énergie dans la section de mesure: } \\
& \quad \pm 0,2 \% ;
\end{aligned}
$$

(1) - Compte non tenu cependant du fait que les pertes d'énergie imputable à l'usure sont nulles pendant les périodes d'apports excédentaires. 
- instabilité de la température dans le temps : fraction de $\%$.

La correction due à $\alpha$ (coefficient thermodynamique) est la plus importante, mais c'est un élément de calcul. D'autre part, on peut éliminer ou réduire les corrections: dépassant éventuellement $1 \%$ en choisissant des conditions expérimentales meilleures : nuit, pour éviter les variations de température, section éloignée de la turbine pour éviter l'hétérogénéité de l'énergie dans la section. En général, toutes les corrections ne sont pas dans le mème sens, el leur ensemble dépasse rarement $1 \%$.

Pour M. Bergenon, l'application de la nethode atux pompes se heurtera toujours à la difficulte de choisir une prise de pression au refoulement suffisamment ‘loigné de la pompe pour éliminer les rotations mais restant en amont des organes d'obturation susceptibles d'introduire des pertes supplémentaires.

M. WrLtM et M. FenRY précisent que les organes d'obLuration ne sont pas gênants au point de vue de la méthode thermométrique elle-même, car l'élévation de température qu'ils introduisent dans le numérateur do l'expression « rendement » est compensée par une baisse de pression : c'est seulement dans la mesure de la chute ou de l'élévation, c'est-à-dire dans le dénominateur qui est commun avec celui de la méthode classique, que ces organes crèent une difficulté.

Sur la demande de M. Bergeron, M. Wiltm précise que dans un cas particulier (mesure de rendement d'unc pompe) l'écart maximum entre les rendements relevés point par point sur un diamètre et celui résultant de l'extrapolation à toute la section de la répartition de l'énergie relevée sur ce diamètre était de $1,2 \%$; la seetion de mesure était située d'une façon particulièrement défavorable, immédiatement à la sortie de la pompe.

M. Darrieus pense que la méthode serait moins incertaine si on prenait pour terme de comparaison une turbine de rendement unilé au lieu d'une turbine fictive de rendement zéro. Dans le premier cas, en effet, la mesure porterait sur $1-\eta$ au lieu de porter sur $\eta$ et la précision serait, en principe, 10 fois plus grande.

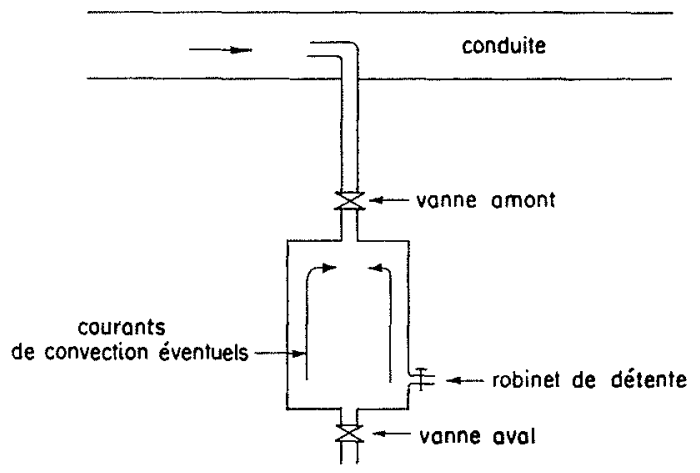

Pour y arriver, précise M. Darrieus, on peut imaginer que l'on prélève dans la conduite forcée un certain volume pour remplir, en principe (sauf correction) au niveau du canal de fuite, un sas, muni de deux vannes, une vanne amont et une vanne aval. Ayant purgé le sas et ayant rempli le sas d'eau dans les conditions de la conduite forcée, c'est-à-dire en évitant tout laminage par la connexion amont, on fermera la vanne aval, puis la vanue amont; enfin un troisième robinet ou, simplement, l'entrouverture de la vanne aval, assurera la détente par l'échappement d'une quantité très petite, correspondant à la compressibilité de l'ean et à l'élasticité du récipient. Toutes les pertes de détente seront concentrées dans ce petit volume évacué, tandis que le volume restant dans le sas aura subi une détente purement adiabatique, dont la représentation dans le diagramme entropique est une droite verticale ou isentropique.

Nous n'aurons plus qu'à comparer, avec la température dans le canal de fuite, celle de cette quantité d'eau restant ainsi dans le sas; et comme il s'agit d'eau à la pression atmosphérique dans des conditions voisines de celles du canal de fuite, on peut penser a priori que la détermination de l'écart de température sera plus précise que dans les conditions actuelles, a condition que la mesure dans le sas s'effectue dans une région centrale assez éloignée des parois, des couches limites et de tout ce qui peut être troublé par l'extérieur, par exemple par un apport de chaleur, déterminant des courants de convection qui remontent le long des parois. 'Tandis que, pour la partie centrale, on peut escompter que l'évolution en est demeurée adiabatique, comme dans un cylindre idéal où on provoque un petit mouvement du piston. Ici, ce n'est pas un piston qui se déplace, c'est une quantité d'eau qui sort, mais, pour la majeure partie, celle qui demeure dans le sas sans contact direct avee l'extérieur, la détente est vraiment isentropique, ou sans pertes.

La méthode thermoélectrique serait ainsi rendue, non seulement beaucoup plus précise, mais encore plus directe, et surtout affranchie des incertitudes qui affec tent la connaissance, nécessaire dans la méthode actuelle bien qu'encore imparfaite, des propriétés thermoelastiques de l'eau, notamment dans les conditions réelles de l'essai (gaz dissous, etc.).

M. le Président remercie M. Willa et propose que la Division technique générale d'E.D.F. réfléchisse au problème posé par M. Darrieus.

\section{Réponse de M. WiLlm à l'intervention de M. Danrieus, reçue après la séance}

Etant donné l'heure déjà tardive, je n'ai pas voulu sur le moment entrer dans une discussion qui eut d'autant plus risqué de se prolonger que la remarque de M. Darrieus paraissait a priori fort judicieuse daus sa nouveauté.

Cependant, je tiens, conformément d'ailleurs à l'invitation de M. le Président, à faire connaître, après réflexion, mon avis sur la question soulevée par M. Darrieus.

Je ferai observer tout d'abord que notre mode expérimental ne consiste pas, contrairement à ce que pense celui-ci, à prendre pour terme de comparaison une turbine de rendement nul : c'est plutôt la méthode par détente totale pratiquée par M. Porrson qui procéderait ainsi.

D'ailleurs, parler au sujet de notre modalité expérimentale de turbine de référence de rendement 1 ou do rendement 0 ne peut que fausser les idées : il faut dire que nous comparons deux niveaux d'énergie en nous plaçant, pour des raisons de commodité expérimentale et de précision, dans des conditions telles que la différence entre ces niveaux ne se manifeste que par une différence de pression à l'exclusion de toute différence de température.

En ce qui concerne la modalité proposée par M. DARRIEUs, sa justification reposerait sur deux hypothèses :

a) La précision absolue d'une mesure portant sur les pertes est supérieure à celle d'une mesure portant sur l'énergie utilisée, du fait que les premières représentent une énergie 10 fois plus faible que les secondes (étant sous-entendu que les erreurs relatives sont les mêmes dans les deux cas).

b) Il est plus exact d'inclure les phénomènes thermodynamiques dans l'expérimentation que d'introduire un coefficient correcteur calculé.

L'idée de mesurer les pertes (en mesurant la différence de température entre canal de fuite et conduite forcée) est ancienne, c'était même l'idée de base de M. Polrsox; 
il ne l'a jamais expérimentée et de toute façon les résultats qu'il eût ainsi obtenus eussent été erronés en raison de la méconnaissance des phénomènes thermoélastiques. Remarquons que le principe de notre expérimentation s'accommoderait aussi bien d'une mesure directe des pertes : il nous suffirait de mesurer au moyen d'un manomètre différentiel la différence de pression entre la conduite forcée et l'enceinte de mesure du détendeur. Nous ne le faisons pas, parce que la mesure en serait compliquée sans aucune augmentation corrélative de précision : en effet, la cause d'erreur la plus importante est constituée par le seuil de sensibilité de la mesure thermométrique; ce seuil correspond à une erreur absolue, dont l'incidence sur le rendement est identique quelle que soit la méthode entployée. Ceci mis à part, il faut porter au passif de la modalité de M. Darrieus l'erreur relative qu'il commettrait sur la mesure de différence de température, certainement supérieure à celle que l'on commet sur une mesure de pression.

En ce qui concerne le deuxième argument de la modalité proposée par M. Darrieus, et dont l'application constitue le deuxième temps de son expérimentation et la véritable originalité, je ferai ressortir que le refroidissement du fait de la détente obéit à des lois simples, et l'on ne voit pas bien la nécessité de renouveler $\grave{a}$ chaque fois les aléas de sa mesure; il est plus expéditif et plus exact de faire intervenir on un résultat d'expérience acquis une fois pour toutes, ou une valeur calculée. Les physiciens qui ont voulu mesurer directement en laboratoire l'effet thermoélastique en réalisant une détente dans des conditions qui ressemblent beaucoup à celles proposées par M. Darrieus, ont trouvé des chiffres franchement aberrants (Pushin et Grebenschikow), ou très dispersés (Joule). Les valeurs calculées à partir de constantes physiques plus faciles à mesurer avec précision (coefficient de dilatation ou de compression) sont certainement plus exactes, et c'est bien la solution que nous avons adoptée.

Je signalerai en outre quelques sujétions qui sont propres à la modalíté de M. Darrieus, et dont l'expérience nous a appris l'importance : le caractère discontinu de la mesure; les délais nécessaires pour le soutirage, d'une part (lent pour éviter les phénontienes dynamiques), puis la mise en équilibre thermique de la sonde après détente, toutes choses favorisant les échanges de calories avec l'extérieur; le fait que pour effectuer une mesure de température au millième de degré, une convection assez importante du liquide est nécessaire, convection pratiquement contraire à l'esprit de l'expérimentation, par définition statique, de M. Darrieus (c'est là qu'il faut sans doute chercher la raison des échecs des mesures directes du refroidissement adiabatique dont je parlais plus haut); enfin, nécessité d'étalonnage des jauges thermométriques.

On connaît, par contre, les avantages et la simplicité de notre expérimentation : soutirage continu avec poss1bilité de débit assez important, pas de mesures de température mais simple égalisation, donc pas de tarage des jauges; deux mesures de pression effectuées avec la même presse à poids mort l'une représentant le numé- rateur, l'autre le dénominateur de l'expression du rendement, ce qui exclut la nécessité d'un étalonnage ou d'un tarage rigoureux de cette presse. Quant aux incertitudes sur les valeurs exactes des corrections thermoélastiques, j'ai fait ressortir que leur calcul à partir du coefficien de dilatation et de compressibilité était certainement plus exact que leur mesure directe et chiffré l'erreur maxima à eraindre sur le rendement à $0,3 \%$, valeur très probablement fortement surestimée.

Ma conclusion est que la modalité préconisée par M. Darrivus ne pourrait en aucun cas être, comme il l'estime, 10 fois plus précise que celle que nous appliquons : tout au contraire, on se trouverait en présence de sources d'erreurs plus importantes avec des complications et des difficultés pratiques supplémentaires, et sans doute un matériel plus volumineux.

Tout ce qui précède ne signifie pas que nous considérons comme idéale la forme actuelle de notre expérimentation : en fait, nous cherchons toujours à perfectionner notre appareillage en diminuant l'incidence des apports de calories, en augmentant la stabilitè et la sensibiliti du dispositif thermométrique, en rendant lensemble encore plus maniable et plus léger. Mais nous ne voyons pas quel mode opératoire nouveau permettrait d'un seul coup un gain substantiel sur tous ces tableaux. Je dirai même qu'il est utopique d'escompter par cette voie une amélioration importante de la précision : en eifet, sous sa forme simple et condensée actuelle, la méthode permet couramment une précision supérieure à $0, \tilde{0} ;$ ol les incertitudes résiduelles les plus gênantes ne seront à la limite imputables ni aux procédés ni à l'appareillage, mais à des phénomènes parasites indépendants die la méthode (gradients d'énergic dans les sections, variations de température, etc.).

Enfin, n'oublions pas que la méthode thermodynamique n'est pas que précise : elle prètend aussi à la commodité et à la légèreté. A ce titre, et sur le plan industriel, toute amélioration d'une précision déjà excellente n'est plus justifiée si elle doit s'accompagner d'une compli. eation ou d'un alourdissement.

\section{Remurques de M. Darkieus}

à la suite de la réponse de M. Willa

La méthode que nous avons proposée a pour but essentiel de réduire dans une proportion importante l'incidence sux le résultat de la mesure des corrections thermoélastiques. Il se peut que la mise au point révèle des difficultés d'application qui en ruinent l'intérêt pratique, et il n'est pas question d'opposer notre proposition sous la forme rudimentaire que nous avons esquissée à la méthode actuelle que M. WiLLM et ses collaborateurs ont portée déjà à un haut degré de perfection. Mais quel que doive être l'avenir pratique de notre proposition, nous persistons à penser qu'elle comporte certains avantages de principe et que sa conception contribue à faire apparaître plus clairement le rôle des corrections thermoélastiques, comme le montre la considération d'un diagramme de Mollier (entropie-enthalpie) pour l'eau, sur l'établissement duquel nous espérons avoir loceasion de revenir à une prochaine occasion. 